\title{
SENSE: a fast RNA-seq library preparation protocol with superior strand specificity
}

\author{
The SENSE mRNA-seq library preparation kit generates ready-to-sequence libraries from low amounts \\ of total RNA within $4 \mathrm{~h}$. SENSE libraries are significantly more strand specific (>99.9\%) than leading \\ alternatives and are virtually free of rRNA contamination. The protocol does not rely on RNA or CDNA \\ fragmentation or additional kits for rRNA depletion, library amplification or size selection.
}

Introduction

With the rapid development of next-generation sequencing technologies, RNA-seq has become the method of choice for transcriptome analysis. Most next-generation sequencing platforms require library construction prior to sequencing, and several current mRNA-seq library preparation protocols conserve the strand orientation of transcripts. This allows the specific assignment of reads to one strand of the genome and enables the discovery and quantification of antisense transcripts as well as overlapping genes transcribed from opposite strands.

However, current RNA-seq protocols do not provide sufficient strand specificity; reads mapping to both strands are generated during library preparation through a number of mechanisms, and this background noise obscures the detection of antisense transcripts.

SENSE provides unprecedented strand specificity, allowing the detection and accurate quantification of antisense transcripts by minimizing false-positive reads.

\section{The SENSE workflow}

SENSE is a complete RNA-to-sequencer solution that requires no additional kits (Fig. 1). It contains an integrated poly(A) selection on magnetic beads that outperforms existing methods. Libraries are then generated with a random priming approach using Lexogen's strand displacement stop/ligation technology.

In a single-tube reaction, starter/stopper heterodimers containing platform-specific linkers are hybridized to the mRNA, where the starters serve as primers for reverse transcription. Reverse transcription terminates upon reaching the stopper from the next heterodimer, at which point the newly synthesized cDNA and the stopper are ligated while still bound to the RNA template. No time-consuming fragmentation step is required, eliminating the need for mechanical

\section{Courtney Nadeau \& Alexander Seitz}

Lexogen $\mathrm{GmbH}$, Vienna, Austria. Correspondence should be addressed to A.S (alexander.seitz@lexogen.com). shearing or additional enzymes. Library size is determined by the protocol itself, not with post hoc size selection. Libraries can be sequenced with standard single-end or paired-end reagents. SENSE supports multiplexing with in-line barcodes that can be introduced with no additional effort and do not require a third barcode-specific sequencing read. One person can prepare 8 sequence-ready libraries starting from total RNA within $4 \mathrm{~h}$ with minimal equipment and no additional kits.

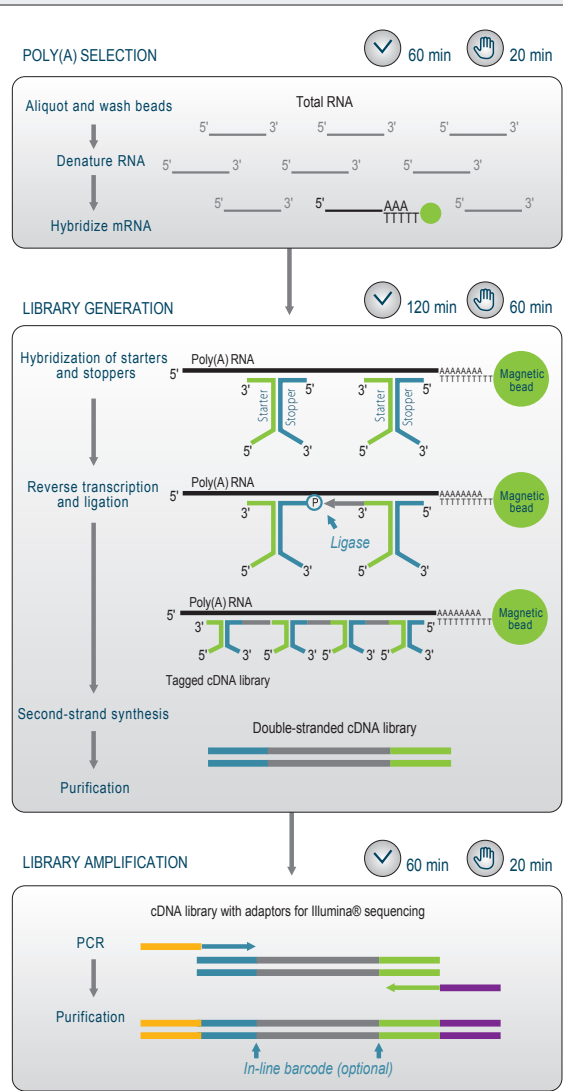

Figure 1 | Overview of the SENSE protocol. 
Table 1 | Summary of sequencing results.

\begin{tabular}{|c|c|c|c|c|}
\hline Total RNA & Strand specificity ${ }^{a}$ & False antisense reads ${ }^{b}$ & $\begin{array}{c}\text { Sense reads } \\
\text { (genome-wide)c }\end{array}$ & rRNA content ${ }^{d}$ \\
\hline $2 \mu \mathrm{g}$ & $99.989 \pm 0.008 \%$ & $0.011 \pm 0.008 \%$ & $99.888 \pm 0.002 \%$ & $0.00014 \pm 0.00003 \%$ \\
\hline $1 \mu \mathrm{g}$ & $99.990 \pm 0.004 \%$ & $0.010 \pm 0.004 \%$ & $99.832 \pm 0.017 \%$ & $0.00036 \pm 0.000005 \%$ \\
\hline $500 \mathrm{ng}$ & $99.996 \pm 0.001 \%$ & $0.004 \pm 0.001 \%$ & $99.857 \pm 0.036 \%$ & $0.00027 \pm 0.00002 \%$ \\
\hline $50 \mathrm{ng}$ & $99.965 \%$ & $0.035 \%$ & $99.779 \%$ & $0.00044 \%$ \\
\hline
\end{tabular}

aNumber of reads mapping to ERCC genes in the sense direction divided by total number of reads mapped to the ERCC genome. bNumber of antisense reads mapping to ERCC transcripts divided by the total

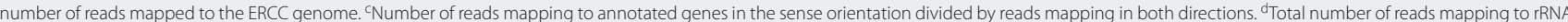
genes divided by the number of reads mapped to any annotation.

\section{Experiment}

We generated multiplexed SENSE libraries from various amounts of Universal Human Reference RNA (Agilent Technologies) with ERCC spike-in RNA controls ${ }^{1}$ (Ambion Inc.) and sequenced these on an Illumina ${ }^{\circledR}$ HiSeq using 100 bp single-end reagents. Pass filter reads (178 million) were de-multiplexed and mapped to the human and ERCC reference genomes. Cytoplasmic rRNA content was very low, indicating efficient poly(A) mRNA selection (Table 1).

The ERCC spike-in transcripts allow the accurate calculation of strandedness as all antisense ERCC reads can be considered false positives introduced during library preparation. In contrast, genomewide calculations of strandedness are conflated by true antisense transcription $^{2}$. Strand specificity was therefore calculated based on ERCC data only and was exceptionally high with all amounts of input RNA (Table 1).

With the $99.99 \%$ strand specificity determined from ERCC data, we detected 2,904 antisense transcripts in the pooled dataset. As the abundance of a sense transcript is often several orders of magnitude higher than its corresponding antisense, signal from actual antisense transcripts can easily drown in false-positive noise generated due to low strand specificity. To examine the relationship between strandedness and antisense detection, we implied lower levels of strand specificity to the data and recalculated the number of antisense transcripts detected (Fig. 2).

Decreasing strand specificity from $99.99 \%$ to $99 \%$ greatly reduced sensitivity and only half as many $(1,506)$ antisense transcripts could be detected. The large loss of sensitivity with even 99\% directionality highlights the extreme importance of maintaining high strand specificity when analyzing antisense gene expression.

One of the primary causes of antisense background and low strand specificity is spurious second-strand synthesis during reverse transcription. Lexogen's strand displacement stop/ligation technology effectively suppresses this background reaction, providing SENSE libraries with the exceptionally high strand specificity required for the detection and accurate quantification of antisense transcripts.

\section{Conclusions}

The SENSE mRNA-seq library preparation protocol is a fast all-in-one solution for the production of strand-specific mRNA-seq libraries starting from total RNA. The integrated poly(A) selection virtually eliminates rRNA contamination without relying on additional selection or depletion protocols. SENSE libraries exhibit exceptional strand

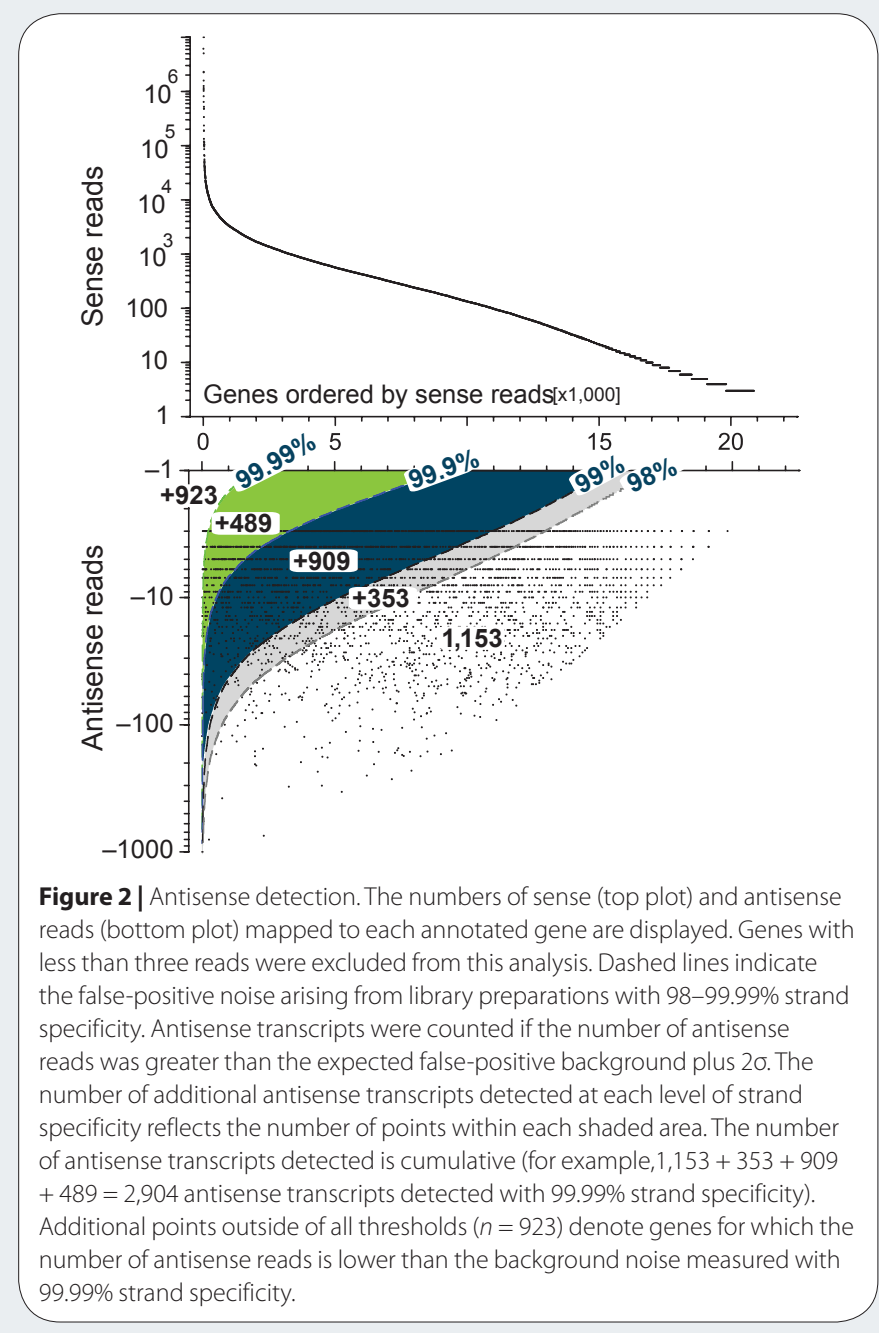

specificity, reduce experimental noise and empower the detection of antisense transcription.

\section{Acknowledgements}

Experiments were planned by Pamela Moll and conducted by Christoph Stüttler, Andrea Czollner and Musashi Tsujita. Bioinformatics analysis was provided by Michael Ante.

1. Baker, S. et al. External RNA Controls Consortium: a progress report. Nat Methods 2, 731-734 (2005).

2. Jiang, L. et al. Synthetic spike-in standards for RNA-seq experiments. Genome Res. 21, 1543-1551 (2011).

This article was submitted to Nature Methods by a commercial organization and has not been peer reviewed. Nature Methods takes no responsibility for the accuracy or otherwise of the information provided. 